\title{
mTOR inhibition: a double-edged sword in patients with COVID-19?
}

\author{
Morteza Ghasemnejad-Berenji ${ }^{1}$
}

Received: 16 January 2021 / Accepted: 22 January 2021 / Published online: 1 February 2021

(c) Japan Human Cell Society 2021

\begin{abstract}
The current COVID-19 is one of the deadliest pandemics in recent decades. In the lack of a specific treatment for this novel infection, knowing the role of cell signaling pathways in the pathogenesis of this infection could be useful in finding effective drugs against this disease. The mammalian or mechanistic target of rapamycin (mTOR) is an important cell signaling pathway that has important role in the regulation of cell growth, protein synthesis, and metabolism in reactance to upstream signals in both pathological and normal physiological conditions. Recently, some researchers have suggested the therapeutic potential of mTOR inhibitors such as rapamycin against COVID-19. However, it is important to consider the role of activation of this pathway in controlling immune system response against viral activity in drug repositioning of rapamycin and other mTOR inhibitors in SARS-CoV-2 infection.
\end{abstract}

Keywords Cell signaling $\cdot$ COVID-19 $\cdot$ Immune system $\cdot$ mTOR $\cdot$ Rapamycin $\cdot$ SARS-CoV-2

$\begin{array}{ll}\text { Abbreviations } \\ \text { COVID-19 } & \text { Coronavirus disease 2019 } \\ \text { mTOR } & \text { Mechanistic target of rapamycin } \\ \text { mTORC } & \text { Mechanistic target of rapamycin complex } \\ \text { SARS-CoV-2 } & \begin{array}{l}\text { Severe acute respiratory syndrome corona- } \\ \text { virus } 2\end{array} \\ \text { TNF- } \alpha & \text { Tumor necrosis factor alpha }\end{array}$

The mechanistic (previously referred to as mammalian) target of rapamycin (mTOR) pathway which is a central regulator of cell metabolism, survival, proliferation, and growth is one of the most important cellular pathways. It senses both extracellular and intracellular signals to regulate the lipids metabolism, transcription, protein synthesis, and autophagy. mTOR is one of the main members of the phosphatidylinositol 3-kinase-related kinase family of protein kinases which is a crucial component of at least two distinct multiprotein complexes, mTOR complex 1 (mTORC1) and mTOR complex 2 (mTORC2), which control different signals to regulate different cellular functions. The

Morteza Ghasemnejad-Berenji morteza.ghasemnejad@yahoo.com; ghasemnejad.m@umsu.ac.ir

1 Department of Pharmacology and Toxicology, Faculty of Pharmacy, Urmia University of Medical Sciences, Nazlou Road, Urmia, Iran rapamycin-sensitive mTORC1 mainly functions as a redox/ nutrient/energy/sensor and controls organelle biogenesis, lipid metabolism, and protein synthesis. The rapamycin-insensitive mTORC2 is a key regulator of the actin cytoskeleton, metabolism, and cell survival [1]. The latest published review articles suggest the therapeutic potential of mTOR inhibitors such as rapamycin against COVID-19 [2-4]. Due to the important role of the mTOR pathway in various cellular processes, it is not astonishing that this pathway induces the development and response of different immune cells. mTOR regulates the gene expression of myeloid immune cells to control their cytokine expression and migration. Inhibition of mTORC1 promotes the autophagy of macrophages and enhances the T-cell stimulatory activity of dendritic cells [5]. mTORC2 controls cell polarity and chemotaxis of mast cells and neutrophils [6]. On the other side, activation of mTORC1 stimulates the secretion of IL-15 which regulates the rate of proliferation and cytotoxicity acquisition of NK cells. It has been indicated that early B-cell production in germinal centers is suppressed by mTOR inhibitors. Therefore, this pathway is important in proliferation and development of B cells. Furthermore, mTORC1 regulates BCL6 expression to determine the fate of B cells in the human germinal center. In fact, after B-cell activation, the inhibition of $\mathrm{mTORC} 1$ decreases the populations of antigen-specific memory B cells [7]. Therefore, it is predictable that treatment with rapamycin prevents B-cell growth and inhibits murine B-cell proliferation induced by 
anti-IgM [8]. Considering the effects of mTOR inhibitors in blocking the development of memory B cells, patients with SARS-CoV2 infection treated with mTOR inhibitors are anticipate to have decreased early cross-reactive antibody production and thus less antibody-dependent enhancement. In addition, $\mathrm{mTORC} 1$ and $\mathrm{mTORC} 2$ activation can induce anti-inflammatory cytokine IL-10 production to prevent apoptosis [9]. mTOR activation can also increase the activity of anti-inflammatory cytokine IL-10 and inhibit the proinflammatory cytokine TNF- $\alpha$. Furthermore, mTOR activation has been reported to be essential for reducing influenza A virus replication, anti-hepatitis $\mathrm{C}$ activity, promoting natural killer cell effector function, and be required for type-1 interferon response to enhance seasonal influenza vaccination clinical effectiveness [10]. Therefore, it is important to consider the role of mTOR activation in regulating immune system response and its antiviral activity in drug repositioning of rapamycin and other mTOR inhibitors in SARSCoV-2 infection and it should be kept in mind that mTOR inhibitors could act as a double edge sword in patients with COVID-19. Therefore, their safety and effectiveness against SARS-CoV-2 infection should be studied in further future studies.

Author contributions M. Ghasemnejad-Berenji, writing the manuscript.

Data availability Data sharing is not applicable to this article as no new data were created or analyzed in this study.

\section{Compliance with ethical standards}

Conflict of interest There is no conflict of interest.

\section{References}

1. Laplante M, Sabatini DM. mTOR signaling at a glance. J Cell Sci. 2009;122(20):3589-94.

2. Husain A, Byrareddy SN. Rapamycin as a potential repurpose drug candidate for the treatment of COVID-19. Chemico-Biol Interact. 2020;331:109282.

3. Omarjee L, Janin A, Perrot F, Laviolle B, Meilhac O, Mahe G. Targeting T-cell senescence and cytokine storm with rapamycin to prevent severe progression in COVID-19. Clin Immunol (Orlando, Fla). 2020;216:108464.

4. Zheng Y, Li R, Liu S. Immunoregulation with mTOR inhibitors to prevent COVID-19 severity: a novel intervention strategy beyond vaccines and specific antiviral medicines. J Med Virol 2020;92(9):1495-1500.

5. Yang H, Wang X, Zhang Y, Liu H, Liao J, Shao K, Chu Y, Liu G. Modulation of TSC-mTOR signaling on immune cells in immunity and autoimmunity. J Cell Physiol. 2014;229(1):17-26.

6. Halova I, Draberova L, Draber P. Mast cell chemotaxis-chemoattractants and signaling pathways. Front Immunol. 2012;3:119.

7. Weichhart $T$, Hengstschläger M, Linke M. Regulation of innate immune cell function by mTOR. Nat Rev Immunol. 2015;15(10):599-614.

8. Ye L, Lee J, Xu L, Li W, Hale JS, Tan WG, Wu T, Davis CW, Ahmed R, Araki K. mTOR promotes antiviral humoral immunity by differentially regulating CD4 helper T cell and B cell responses. J Virol 2017;91(4):e01653-16.

9. Katholnig K, Linke M, Pham H, Hengstschläger M, Weichhart T. Immune responses of macrophages and dendritic cells regulated by mTOR signalling. London: Portland Press Ltd.; 2013.

10. Maiese K. The mechanistic target of rapamycin (mTOR): novel considerations as an antiviral treatment. Curr Neurovasc Res. 2020;17(3):332-7.

Publisher's Note Springer Nature remains neutral with regard to jurisdictional claims in published maps and institutional affiliations. 